\title{
IVR Test \& Survey: A computer program to collect data via computerized telephonic applications
}

\author{
LOUIS H. JANDA, MICHAEL JANDA, and ERIC TEDFORD \\ Old Dominion University, Norfolk, Virginia
}

\begin{abstract}
Several studies have demonstrated the advantages of using interactive voice response (IVR) technology to collect self-report data from research participants and recipients of psychological/medical services. IVR allows participants to phone a computer and respond to recorded questions by pressing the appropriate touch-tone keys on their telephone. Because this technology offers substantial benefits in terms of cost and efficiency, it is surprising that it has not been more widely utilized by researchers and practitioners. Along with the automation of the administration and scoring of tests or surveys, IVR provides for questioning to be adapted to the participants' responses. One possible explanation for the failure to exploit this technology is the absence of easy-to-use software that does not require programming knowledge. This article describes IVR Test \& Survey, a program to facilitate the administration, scoring, and analysis of information collected with the use of IVR technology.
\end{abstract}

The power of interactive voice response (IVR) technology is evidenced by its wide use in business and industry. This technology allows people to interact with a computer system by using their touch-tone telephones. It has become routine, for example, for people to use their touch-tone telephone to call a computer to find their bank balances, to check on their retirement accounts, or, at many universities, to register for classes for the coming semester. Similarly, researchers and practitioners could use this technology to automate the collection of survey or questionnaire responses from participants or clients.

Over the past few years, a few researchers have used IVR to collect such data. Mundt, Perrine, Searles, and Walter (1995) asked 51 people to call their computer to provide information about daily alcohol use for 51 people over a period of 112 days. The researchers observed that this technology not only facilitated the collection of the data, but made it possible to recover data that might have been lost had more traditional methods been used. If a participant failed to make a scheduled call, this could be discovered within hours, whereas it would have taken several days had the participant failed to mail a questionnaire.

Baer, Brown-Beasley, Sorce, and Henriques (1993), who used IVR to perform assessments of 18 OCD patients, found that the results were highly correlated with information collected via paper-and-pencil self-report and with information collected by a research assistant over the phone.

Correspondence concerning this article should be addressed to L. H. Janda, Department of Psychology, Old Dominion University, Norfolk, VA 23529 (e-mail: ljanda@odu.edu).The program is owned by the first author and the cost of the software at the time of this writing is \$100. More information can be obtained from L. H. Janda at janda@ picusnet.com.
Thus, IVR allowed for greater efficiency with no apparent sacrifice in reliability or validity.

As a final example, Baer et al. (1995) used this technology to perform assessments of depression of 1,812 people, a task that would be quite costly and time-consuming with the use of traditional methods.

Given the advantages that IVR technologyoffers researchers and practitioners, it is surprising that it has not been used more widely. Perhaps one reason for this failure is the absence of software that can be used by nonprogrammers to develop and administer applications. To our knowledge, there is no program available that is designed specifically for the administration of tests, questionnaires, or surveys in this manner.

In the studies that have been published using this technology, researchers have selected one of two available options. The first is to use a development package to create a specific application. Commercial packages typically make it possible to develop a variety of IVR applications (e.g., the ability to provide fax-back services or voice mail systems) along with the capacity to develop applications that administer questionnaires or surveys, and a number of these options are available (Warren, 1994). For example, Mundt et al. (1995) used a package sold by EASE, and the current authors have used a similar package sold by Artisoft. These packages do require programming knowledge in either Visual Basic or Visual C++, and the cost is based on the number of phone lines to be used in the application. For four phone-line capability, EASE costs $\$ 2,800$ and Artisoft's package is $\$ 1,400$. A relatively new package that requires no programming knowledge is offered by VOS, and the cost of this package, including the necessary hardware for four lines, is $\$ 3,300$. These packages must be integrated with a database program that stores the voice 
prompts (i.e., questionnaire or survey items) and the responses of callers. Also, these development packages do not provide for the scoring of responses or for any statistical analyses. These tasks must be accomplished by exporting the data to a statistical package or spreadsheet.

The second option available to researchers who wish to use IVR technology is to contract with a company that develops and administers applications, as did Baer et al. (1995). One such company that specializes in providing services relevant to behavioral researchers and practitioners, Health Tech Systems located in Madison, Wisconsin, charges approximately $\$ 40$ per respondent, depending on the test administered, in addition to set-up fees. Clearly, the use of IVR technology currently requires a substantial investment in expertise and time, or, in the absence of these requisites, a substantial budget. Given the enthusiasm that the researchers who have used IVR have for this technology, it would seem likely that if easy-to-use software were available, more researchers would be likely to take advantage of it.

To increase accessibility of IVR to researchers and practitioners, we developed IVR Test \& Survey, a program that facilitates the development of applications that allow participants to call a computer via touch-tone telephone to respond to a test or survey. The program provides for recording the voice prompts, administering the test or survey items, collecting participants' responses, scoring an unlimited number of subscales, and performing a few basic statistical analyses.

\section{Program Operation}

IVR Test \& Survey was written in Visual $\mathrm{C}++6.0$ for IBM-compatible computers utilizing the Microsoft Win- dows 95, Windows 98, or Windows Me operating systems. Several companies sell the hardware necessary to implement IVR, but this program was written to be used with a variety of Dialogic Voice boards, although the initial version has been used only with a Pentium 350 computer equipped with two Dialogic ProLine/2V boards and a similar computer equipped with a single Dialogic $\mathrm{D} / 41 \mathrm{H}$ board. Both configurations allow 4 participants to access the computer at any one time. The manufacturers suggested retail price for the ProLine/2V board is $\$ 514$, and for the $\mathrm{D} / 41 \mathrm{H}$ board $\$ 1,295$, although the street price is at least $10 \%$ lower. The number of boards that can be used is limited by the computer's number of available PCI slots.

IVR Test \& Survey consists of two components: Application Configurator and Application Executer. Application Configurator allows researchers to design their application, record the voice prompts, specify the scoring system to be used with test or survey responses, specify the statistical analyses to be employed, and design the output files. These tasks are accomplished with the use of dialog boxes. The first such box, named the Edit Application, is presented in Figure 1. This example presents participants with a menu that asks them to select either the "Big Five" or a "Customer Survey." Participants provide their input by depressing the appropriate key on their telephone keypad. The program allows for an unlimited number of menu choices, although practical considerations dictate a more modest number.

The program distinguishes two types of participant input. The first type simply provides information, such as an identification number, age, or a number indicating sex. Also, the program is caller ID capable, so researchers have the option of recording the phone number from which the

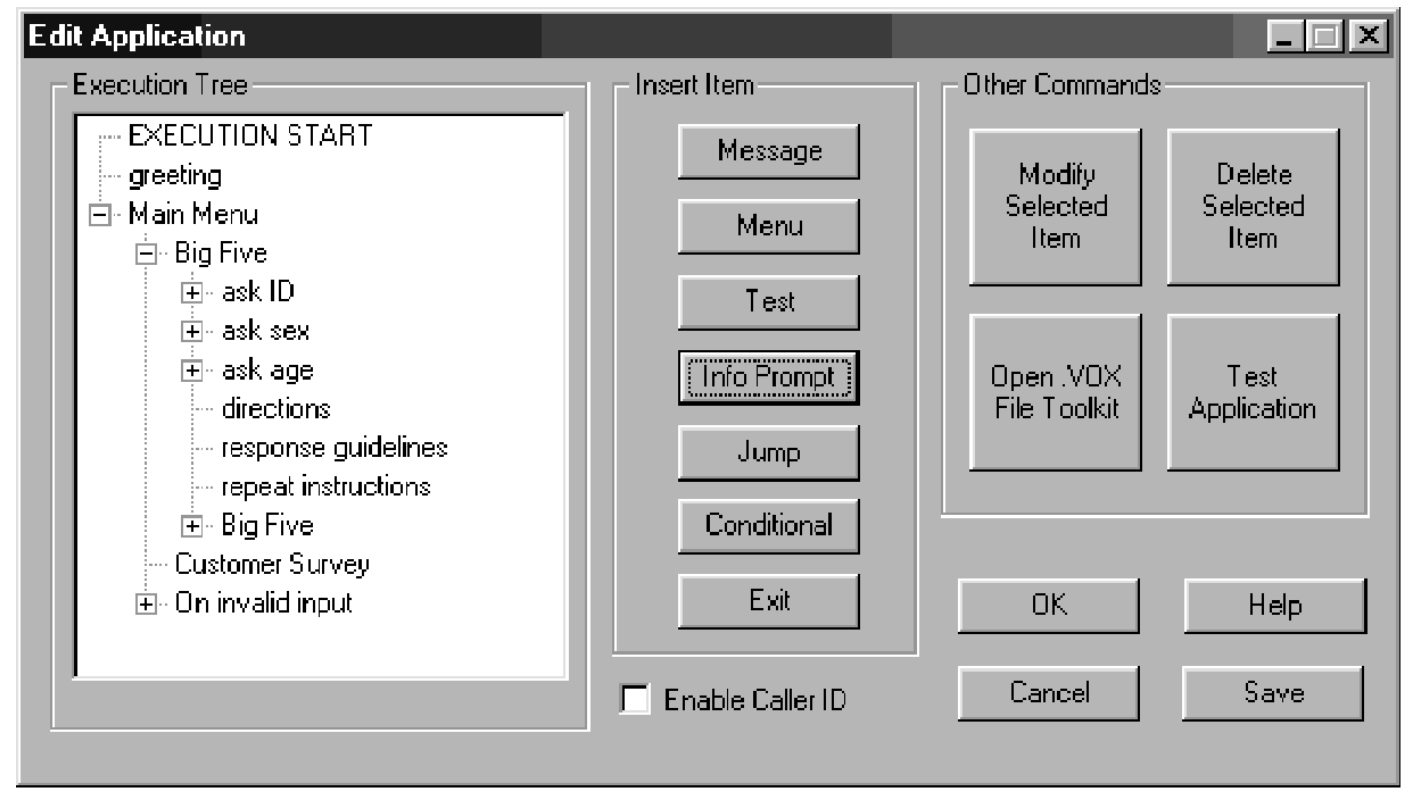

Figure 1. Edit Application dialog box, where one designs the structure of the application. This example reflects two applications: the administration of a 100-item Big Five personality inventory and a "Customer Survey." 
participant is calling. The second type of input consists of responses to test or survey items. With this type of input, researchers must design a scoring system. Although the program was written with Likert-type responses in mind, it is also possible to score items with true/false or multiplechoice formats. Multiple-choice formats may not be desirable, since, in our experience, participants have difficulty remembering all the alternatives. The program does, however, allow participants to request that any item be repeated. Also, if the participant does not respond in a specified period of time, the last item presented will be repeated. If Likert-type responses are used, the program allows researchers to designate appropriate items to be reverse scored. It is possible to derive scores for an unlimited number of items, grouped into an unlimited number of subscales.

The program does provide for a few basic statistical analyses. The dialog box for specifying these can be seen in Figure 2. Sums, means, standard deviations, and variances can be calculated for either individuals or groups. With group data, coefficient alphas and Pearson correlation coefficients can be calculated. With respect to correlations, variables can include information items, test items, subscale scores, or total test scores. It is also possible to perform two-way cross-tabulations using the variables above.

The program allows researchers to design either individual or group output files. Any number of output files can be specified for a particular application. For instance, one file might include participants' responses to all items, while another might include only participants' subscale scores. It is also possible to design individual files for each participant that include their responses to individual items, summary scores, or both. These output files can be exported to spreadsheets or statistical packages for further analyses. Once the researcher is familiar with the software, applications can be prepared quickly. It took us approximately $1 \mathrm{~h}$ to create an application that administered a 100-item version of the Big Five personality test, including the time required for recording the voice prompts. Our experience suggests that an individual who feels comfortable with programs such as SPSS, spreadsheets, or databases would be able to design his or her first application, similar to the one above, using only the instruction manual as a guide in about $3 \mathrm{~h}$.

Once the application has been designed, Application Executer is used to load the appropriate application. This is a simple process that utilizes a dialog box that allows researchers to browse for the intended application and then a second dialog box that asks researchers to specify the phone lines to be used for that application. It is possible to dedicate various phone lines to different applications. Two lines, for example, could be dedicated to the "Big Five," while two remaining lines could be dedicated to the "Customer Survey."

A manual providing samples of applications and discussion of the dialogs accompanies IVR Test \& Survey. While developing an application, one can utilize help files.

In our initial test of the software, we administered Goldberg's (1990) 100-item measure of the Big Five personality inventory to 100 participants who also completed the test in a paper-and-pencil format. Interestingly, a number

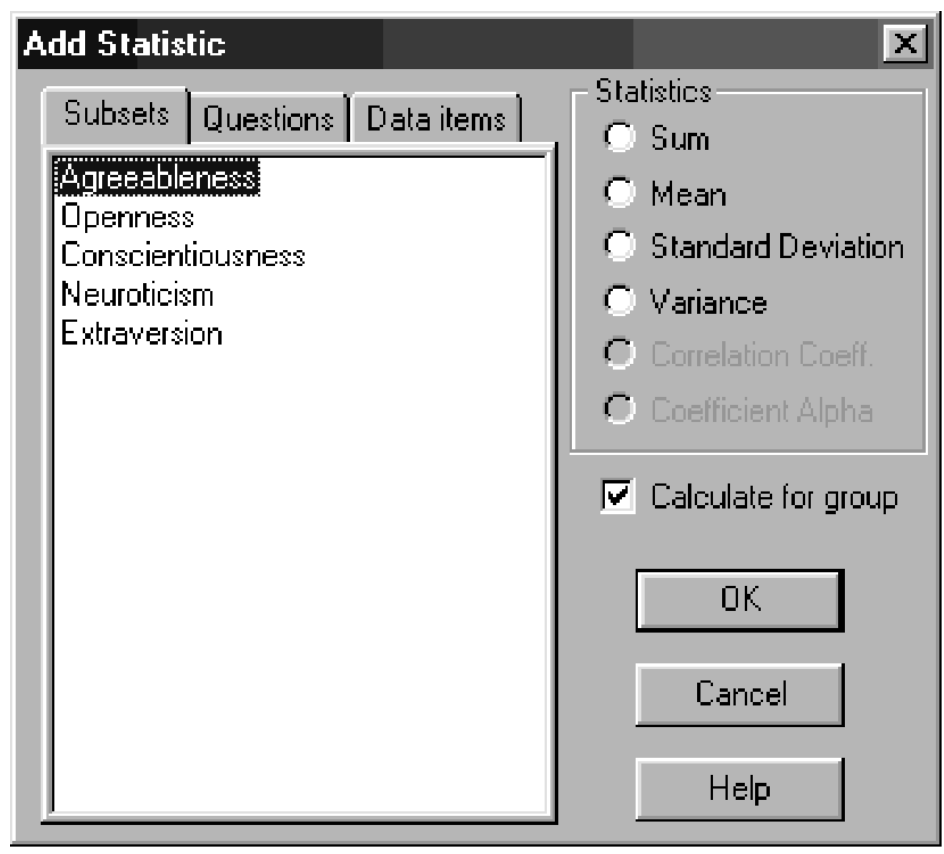

Figure 2. Add Statistic dialog box, where one can specify the statistical calculations to be performed with the five subset scores of Agreeableness, Openness, Conscientiousness, Neuroticism, and Extraversion. 
of participants volunteered that they preferred the telephonic version of the test over the paper-and-pencil version. Subsequently, the first author has used the system to administer questionnaires to his large lecture classes. He has asked the class members (as many as 150 people) to call his computer at any time during the following $36 \mathrm{~h}$. At the next class meeting, students were provided with their scores as well as with descriptive statistics for the class. These brief applications were developed in approximately $20 \mathrm{~min}$.

\section{Availability and Equipment Requirements}

The program can be purchased from the first author. It comes on a compact disc that includes a user manual and a sample application. It has been designed to be used with the Windows 95/98/Me platform on an IBM-compatible computer with at least a Pentium 166 processor and at least $64 \mathrm{MB}$ of RAM. The program requires about $8 \mathrm{MB}$ of hard drive space for installation and for sufficient space to record the voice files. The program requires the computer to be equipped with a Dialogic Voice board.

\section{REFERENCES}

Baer, L., Brown-Beasley, M. W., Sorce, J., \& Henriques, A. I. (1993). Computer-assisted telephone administration of a structured interview for obsessive-compulsive disorder. American Journal of Psychiatry, 150, 1737-1738.

Baer, L., Jacobs, D. G., Cukor, P., O’Laughlen, J., Coyle, J. T., \& MAGRUdER, K. M. (1995). Automated telephone screening survey for depression. Journal of the American Medical Association, 273, 1943 1944.

GolDBERG, L. R. (1990). An alternative "description of personality": The big-five factor structure. Journal of Personality \& Social Psychology, 59, 1216-1229.

Mundt, J. C., Perrine, M. W., Searles, J. S., \& Walter, D. (1995). An application of interactive voice response (IVR) technology to longitudinal studies of daily behavior. Behavior Research Methods, Instruments, \& Computers, 27, 351-357.

WARREN, S. (1994, March/April). Seven great IVR application generators compared. Computer Technology, pp. 64-79.

(Manuscript received July 6, 2000; revision accepted for publication February 5, 2001.). 